\title{
BMJ Open Open-label, multicentre, single-arm trial of monthly injections of depot buprenorphine in people with opioid dependence: protocol for the CoLAB study
}

\author{
Briony Larance, ${ }^{1,2,3}$ Marianne Byrne (1) , ${ }^{1,4}$ Nicholas Lintzeris, ${ }^{5,6}$ \\ Suzanne Nielsen (1D) , ${ }^{1,7}$ Jason Grebely, ${ }^{4}$ Louisa Degenhardt (1) ,1,8 \\ Jeyran Shahbazi, ${ }^{1}$ Marian Shanahan (D) , ${ }^{1}$ Kari Lancaster, ${ }^{9}$ Gregory Dore, ${ }^{4}$ \\ Robert Ali, ${ }^{1,10}$ Michael Farrell, ${ }^{1}$ On behalf of the CoLAB study team
}

To cite: Larance B, Byrne M, Lintzeris $\mathrm{N}$, et al. Open-label, multicentre, single-arm trial of monthly injections of depot buprenorphine in people with opioid dependence: protocol for the CoLAB study. BMJ Open 2020;10:e034389. doi:10.1136/ bmjopen-2019-034389

\section{- Prepublication history and} additional material for this paper are available online. To view these files, please visit the journal online (http://dx.do org/10.1136/bmjopen-2019034389).

Received 18 September 2019 Revised 06 March 2020 Accepted 16 April 2020
Check for updates

(C) Author(s) (or their employer(s)) 2020. Re-use permitted under CC BY-NC. No commercial re-use. See rights and permissions. Published by BMJ.

For numbered affiliations see end of article.

Correspondence to

Dr Michael Farrell;

michael.farrell@unsw.edu.au

\section{ABSTRACT}

Introduction Opioid agonist treatment is effective for opioid dependence and newer extended-release buprenorphine (BUP-XR) injections represent a significant development. The Community Long-Acting Buprenorphine (CoLAB) study aims to evaluate client outcomes among people with opioid dependence receiving 48 weeks of BUP-XR treatment, and examines the implementation of BUP-XR in diverse community healthcare settings in Australia.

Methods and analysis The CoLAB study is a prospective single-arm, multicentre, open-label trial of monthly BUP-XR injections in people with opioid dependence. Participants are being recruited from a network of general practitioner and specialist drug treatment services located in the states of New South Wales, Victoria and South Australia in Australia. Following a minimum 7 days on $8-32 \mathrm{mg}$ of sublingual buprenorphine ( \pm naloxone), participants will receive monthly subcutaneous BUP-XR injections administered by a healthcare practitioner at intervals of 28 days (-2/+14 days). The primary endpoint is participant retention in treatment at 48 weeks after treatment initiation. Secondary endpoints will evaluate dosing schedule variations, craving, withdrawal, substance use, health and well-being, and client-reported treatment experience. Qualitative and costing substudies will examine implementation barriers and facilitators at the client and provider level.

Ethics and dissemination The study has received ethics approval from the St Vincent's Hospital Sydney Human Research Ethics Committee (Ref. HREC/18/SVH/221). The findings will be disseminated via publication in peerreviewed journals, presentations at national and international scientific conferences, and in relevant community organisation publications and forums.

Trial registration number NCT03809143

Protocol identifier CoLAB1801, V.4.0 dated 01 August 2019

\section{INTRODUCTION}

The two main medications used in opioid agonist treatment (OAT), methadone and
Strengths and limitations of this study

- This is an open-label trial with broad eligibility criteria and flexible extended-release buprenorphine (BUP-XR) dosing to more accurately reflect 'realworld' clinical practice compared with previous randomised controlled trials.

- The study will be conducted at a range of healthcare settings, including specialist opioid treatment services and general practice, publicly and privately operated facilities, in metropolitan and regional locations.

- The sample size for the clinical intervention cohort is relatively small with no comparison group; however, rich and detailed participant interview data will be collected to evaluate the impact of BUP-XR on people who are opioid dependent, including those participants who discontinue treatment early.

- This mixed-method protocol (clinical intervention cohort, qualitative research and costing) will focus on the barriers and facilitators to implementation, and will be used to inform models of care, clinical guidelines and training programmes for health providers.

buprenorphine (buprenorphine; \pm naloxone), are well established as safe and effective treatments for opioid dependence. ${ }^{12}$ OAT reduces illicit opioid use, crime, overdose, mortality risk and risk of HIV and hepatitis $\mathrm{C}$ virus (HCV) acquisition. ${ }^{3-5}$ Despite these clear benefits, OAT carries some risks, including adverse events, injection of medication intended for oral/sublingual administration, diversion and overdose. ${ }^{6-8}$

In response to these risks, supervised daily dosing at a specialist clinic or pharmacy is a feature of OAT in many countries ${ }^{1-4}$ at least during the early stages of treatment. 
Attendance for daily dosing is burdensome for both clients and service providers. People in treatment often state that attendance for supervised dosing is restrictive on many aspects of daily life ${ }^{5}$ especially if significant travel time, cost and inconvenience is involved in attending during limited clinic dosing hours. Additionally in Australia, people receiving OAT frequently pay the costs of pharmacy dispensing fees ${ }^{6}$ which is a significant burden for many clients, and also a barrier to treatment access for many who are on income support payments or disability support pensions. ${ }^{6}$ In some Australian jurisdictions, publicly funded specialist treatment services cover the costs of supervised dosing so there is no cost to the client, but this requires substantial staffing resources that may be alternatively utilised for case management or providing health-related and/or psychosocial interventions.

Recently developed extended-release buprenorphine (BUP-XR) injections represent a significant development in OAT. They are administered subcutaneously by a healthcare provider, releasing buprenorphine at a controlled rate over the dosing interval. Currently formulations enable weekly ${ }^{910}$ or monthly administration. ${ }^{9-11}$ Early studies indicate that they provide rapid onset and sustained release of buprenorphine, ${ }^{7812}$ blockade at the mu-opioid receptors minimising the euphorigenic opioid effects of illicit opioid use, ${ }^{13}{ }^{14}$ sustained reductions in illicit opioid use and good treatment retention. ${ }^{1516}$ Oncemonthly BUP-XR injections have been approved in the USA, Canada, Australia ${ }^{17}$ and Europe, and once-weekly BUP-XR injections have been approved in Australia ${ }^{18} 19$ and Europe. ${ }^{20}$

BUP-XR injections are expected to be associated with several potential benefits. Eliminating the need to attend for frequent dosing should increase convenience to clients, and may reduce the costs to clients and providers. Monthly BUP-XR injections may enhance treatment efficacy as a result of fewer missed doses, more consistent buprenorphine plasma levels and improved treatment exposure. ${ }^{21}$ BUP-XR injections delivered by a healthcare professional may also reduce the diversion and use via unintended routes of administration observed with sublingual buprenorphine treatment. ${ }^{22-24}$

There is also substantial client interest in BUP-XR. A recent survey of people who used opioids regularly ${ }^{25}$ found that two-thirds of respondents believed BUP-XR was a good treatment option for them. Interest was high among those currently in OAT as well as those who were not, with no differences according to prior methadone vs buprenorphine experience. ${ }^{25}$ The most common advantages endorsed by participants were less frequent service attendance, 'more time to do other things' and freedom to travel for work or holidays. ${ }^{25}$ Despite these potential advantages, it is important to understand how BUP-XR impacts on client outcomes and whether the outcomes observed with BUP-XR are similar to sublingual buprenorphine despite less frequent contact with treatment services.
Six-month retention on two BUP-XR formulations were $63 \%$ and $69 \%$, respectively, ${ }^{15}{ }^{16}$ in phase III clinical trials. These retention levels are substantially higher than those observed in routine treatment with sublingual buprenorphine,${ }^{26}$ however, the extent to which these trials reflect 'real-world' clinical practice is limited. The doubleblind placebo-controlled or active-controlled designs of these trials required all participants to attend clinics on a weekly basis, which does not reflect the likely routine practice with injections of monthly BUP-XR. Clients were a highly selected group, with a range of exclusions. There are limited data available on the adherence, retention and safety (adverse events) of BUP-XR injections over the longer term, especially the potential for local injection site complications. One open-label safety study with flexible dosing with either weekly or monthly BUP-XR over 48 weeks found high levels of retention (73.6\% at 48 weeks) and the treatment was well tolerated with a safety profile consistent with that for sublingual buprenorphine. ${ }^{27}$ Additional studies conducted over such extended period of treatment and adverse event monitoring are needed.

Implementing BUP-XR in the diverse range of treatment settings in Australia and many other countries also presents challenges. Although specialist publicly funded clinics with onsite pharmacies are available in some jurisdictions, the treatment models in other jurisdictions rely predominantly on primary care prescribers and community pharmacies. Implementing BUP-XR in these diverse settings has important service-level and system-level implications, including the development of new models of care and new procedures for drug storage and administration.

The overarching aims of the Community Long-Acting Buprenorphine (CoLAB) studies detailed in this protocol are:

1. To evaluate the participant outcomes following the implementation of a monthly injection of BUP-XR for the treatment of opioid dependence in communitybased treatment settings with a focus on retention in treatment, opioid and other illicit drug use, adherence with the administration schedule and participants' experiences of the implementation.

2. To develop and document the implementation of a monthly injection of BUP-XR for the treatment of opioid dependence with an emphasis on the feasibility and practical clinical, regulatory and supply issues in settings representative of Australian clinical practice.

\section{METHODS AND ANALYSIS}

\section{Study design}

The CoLAB study is a prospective single-arm, multicentre, open-label trial of monthly BUP-XR (Sublocade) in people with opioid dependence, with qualitative and costing substudies. The primary objective is to examine treatment retention at 48 weeks following initiation of BUP-XR injections in clients with opioid dependence transferred from a stable dose of sublingual buprenorphine. 
A total of 100 people with opioid dependence will be enrolled from seven Australian study site locations, which include a mix of service providers in community settings (eg, primary care based general practitioners) and in specialist clinic settings. Sites were selected to be representative of the range of treatment service models typical in Australia, to facilitate efficient capture of implementation issues. All site principal investigators (PIs) are current OAT prescribers, either addiction specialists $(n=6)$ or general practitioners with experience in delivering OAT $(n=1)$, and were provided additional training by the study team on BUP-XR administration and management. Participant recruitment commenced in May 2019 and is expected to reach completion in late 2019. Qualitative and costing substudies will also be conducted and are described here in brief. The CoLAB study protocol addresses all criteria in the 2013 Standard Protocol Items: Recommendations for Interventional Trials guidelines (online supplementary appendix 1 , and study findings will be reported in accordance with these criteria.

\section{Participant eligibility}

The study population is individuals with opioid dependence who are receiving $8-32 \mathrm{mg}$ sublingual buprenorphine treatment (for at least 7 days), express interest in receiving BUP-XR, and are deemed suitable for treatment with BUP-XR by the site investigator. Eligibility criteria are deliberately broad to allow enrolment of a diverse group of participants. Anyone with significant, medical or psychiatric conditions which would compromise compliance and/or client safety will be excluded. Specific conditions of interest include hepatic disease (Child-Pugh Class B or C), severe renal or respiratory disease, or severe cognitive impairment or psychiatric condition that impairs the ability to provide informed consent (eg, psychosis, delirium, hypomania, severe depression or suicidal ideation). While potential drugdrug interactions for BUP-XR are similar to sublingual buprenorphine, any participant with a history or presence of allergic or adverse response to the ATRIGEL Delivery
System gel polymer component of Sublocade BUP-XR will be excluded. Full eligibility criteria are described in table 1 .

\section{Study schedule}

The study consists of a screening phase (up to 4 weeks); treatment intervention phase (48 weeks) and follow-up at 4 weeks after the last dose of study medication, as depicted in the study schema (figure 1):

(A) Screening: Following informed consent (please refer to online supplementary file 1), screening assessments include: physical assessment, medical history, substance use and related treatment history, urine pregnancy test, urine drug screening, concomitant medication, and eligibility confirmation. (B) Treatment: BUP-XR treatment will be provided over 44 weeks, involving study visits every 4 weeks. Each study visit consists: (1) clinical and safety assessments (2) Administration of BUP-XR injection and (3) research interviews via telephone. (C) Post-treatment follow-up: 4 weeks after the last dose of BUP-XR, for collection of safety event and research interview data.

The procedures conducted at each visit throughout the study are detailed in the CoLAB schedule of assessments (table 2).

\section{BUP-XR treatment}

The protocol allows for participants to receive injections of Sublocade scheduled 28 days apart $(-2 /+14$ days; figure 1). Sublocade contains a buprenorphine base in a precipitation delivery system (ATRIGEL) of biodegradable polylactide-co-glycolide polymer and biocompatible solvent (N-methyl-pyrrolidone), which, in contact with aqueous interstitial fluid, solidifies in the subcutaneous space to form a depot. This solid depot provides sustained release of buprenorphine over a minimum of 28 days through diffusion and polymer degradation. ${ }^{28} 29$

Participants will follow a dosing schedule aligned with the Australian Sublocade product information. ${ }^{11}$ This involves two Sublocade doses of $300 \mathrm{mg}$ at baseline and

Table 1 Participant inclusion and exclusion criteria for the CoLAB study

\section{Inclusion criteria}

Aged 18-65 years

- Opioid-dependent currently receiving treatment

- Has been receiving 8-32 mg sublingual buprenorphine \pm naloxone tablets/film for at least 7 days

- Negative pregnancy test at screening and baseline in females of childbearing potential

\section{Exclusion criteria}

- Currently lactating or pregnant, or of childbearing potential and not willing to avoid becoming pregnant during the study

- History or presence of allergic or adverse response (including rash or anaphylaxis) to buprenorphine or the ATRIGEL Delivery System.

- Significant, medical or psychiatric conditions which would compromise compliance with the protocol and/or client safety. Specific conditions of interest include hepatic disease (Child-Pugh Class B or C), severe renal or respiratory disease, or severe cognitive impairment or psychiatric condition that impairs the ability to provide informed consent.

- Subjects who are currently participating in any other clinical study involving investigational medication(s).

- Inability or unwillingness to provide informed consent or abide by the requirements of the study.

CoLAB, Community studies of Long-Acting Buprenorphine. 


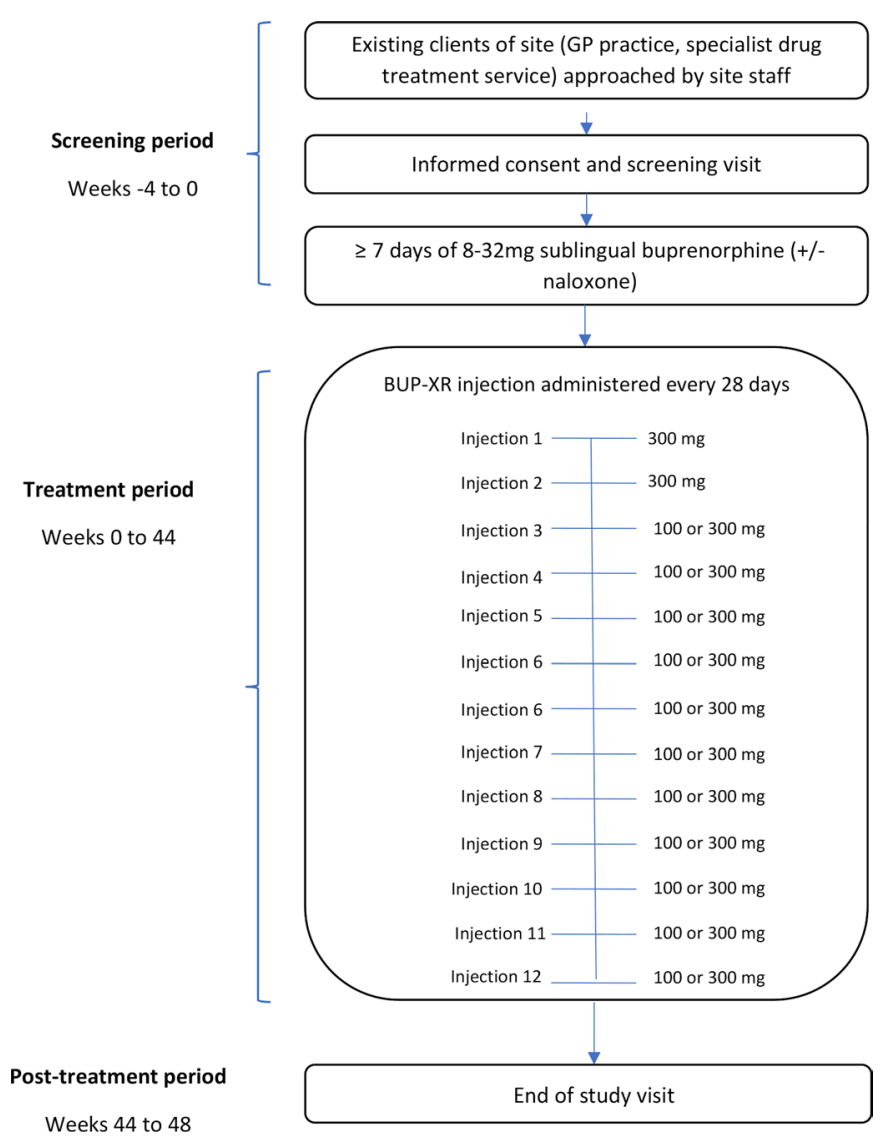

Figure 1 CoLAB study schema. CoLAB, Community studies of Long-Acting Buprenorphine; GP, general practitioner; BUP$\mathrm{XR}$, extended-release buprenorphine.

week 4, reflecting 'loading' doses that elevate plasma buprenorphine levels. Thereafter (doses 3-12), doses are flexible with either 100 or $300 \mathrm{mg}$ every 4 weeks, as decided by the site investigator in consultation with the participant (figure 1). Relevant factors in dose consideration include client craving and withdrawal, client rating of dose adequacy, opioid and other substance use, and adverse events. In general, doses should be maintained (on $100 \mathrm{mg}$ or $300 \mathrm{mg}$ ) if no clinically significant opioid withdrawal, cravings, or dose-related adverse events are identified, and the patient is satisfied with his/her current dose. Doses should generally be reduced from $300 \mathrm{mg}$ to $100 \mathrm{mg}$ if the patient reports dose-related adverse events (eg, sedation, lethargy, headaches, nausea), the patient is seeking to ultimately withdraw from OAT, or the patient reports the dose is 'too high'. Doses should be increased from $100 \mathrm{mg}$ to $300 \mathrm{mg}$ if the patient is not achieving treatment goals (eg, persistent unsanctioned opioid use, withdrawal symptoms or cravings), no doserelated adverse events to buprenorphine are identified, or the patient reports the dose is inadequate and there are no significant clinical safety concerns. All injections will be administered by a medical practitioner or nurse trained to perform the task. The product is administered by subcutaneous injection into the abdominal area only, with the injection site rotated each dose to avoid potential irritation.
Supplemental doses of up to $8 \mathrm{mg}$ daily sublingual buprenorphine for 14 days are permitted, however, after the first 2 BUP-XR doses additional doses will require approval by the study chief medical officer, who is available to provide clinical advice to site staff throughout the study. Treatment is to be discontinued in the event of pregnancy, non-adherence to dosing regimen (more than 56 days between injections), participant removal of the depot or medical deterioration in clinical condition of the participant according to the site PI. Where BUP-XR treatment is discontinued for any reason, individualised advice is provided by the study chief medical officer, typically to reintroduce sublingual buprenorphine based on first principles.

\section{Participant interviews}

Participants will complete interviews via telephone at intervals of 28 days ( \pm 4 days) throughout the treatment period. After the first BUP-XR dose, the interview schedule is independent of the dosing schedule and participants who discontinue treatment will remain in follow-up for interviews. The interviews are structured from a combination of validated tools and additional questions developed by the protocol steering committee (PSC), collecting clientreported withdrawal, craving, substance use, overdose, health service utilisation, work attendance, pain, quality of life, and treatment satisfaction data. The questionnaires included in the interviews are described in table 2. Participants are reimbursed AUD50 per completed interview as compensation for their time.

\section{Outcome measures and analysis}

The primary objective of the CoLAB trial is to examine treatment retention at 48 weeks following initiation of BUP-XR injections at intervals of 28 days $(-2 /+14$ days $)$ in clients with opioid dependence transferred from a stable dose of sublingual buprenorphine. Secondary objectives include evaluation of dosing schedule variations, craving, withdrawal, substance use, health and well-being, and client-reported treatment experience over the treatment period. Study endpoints are described in table 3.

\section{Safety event reporting}

In addition to standard adverse event and serious adverse event (SAE) data collection and reporting to the ethics committee, regulatory authority and Indivior pharmacovigilance, information on adverse events of special interest (AESI) will be collected. AESI include: pregnancy, buprenorphine overdose, severe hepatic impairment, depot removal and severe precipitated withdrawal. A medical monitor independent of UNSW, the CoLAB study and Indivior will review all SAEs for relatedness to BUP-XR and/or study participation. A data safety monitoring board will review interim safety data once the first 20 participants have reached month 6 of the study, with subsequent review commensurate with its risk assessment. 
Table 2 CoLAB study schedule of assessments

\begin{tabular}{|c|c|c|c|c|c|c|c|c|c|c|c|c|c|c|}
\hline \multirow[b]{2}{*}{ Study week } & \multirow{2}{*}{$\frac{\text { Screening }}{-4 \text { to } 0}$} & \multicolumn{12}{|c|}{ Treatment } & \multirow{2}{*}{$\begin{array}{l}\text { Post-T) } \\
48\end{array}$} \\
\hline & & 0 & 4 & 8 & 12 & 16 & 20 & 24 & 28 & 32 & 36 & 40 & 44 & \\
\hline \multicolumn{15}{|l|}{ Clinical assessments } \\
\hline Medical history and physical examination & $\mathrm{X}$ & & & & & & & & & & & & & \\
\hline Substance use and treatment history & $\mathrm{x}$ & & & & & & & & & & & & & \\
\hline \multicolumn{15}{|l|}{ Australian Treatment Outcome Profile (ATOP) X } \\
\hline Past 7 days sublingual buprenorphine dose & & $\mathrm{x}$ & & & & & & & & & & & & \\
\hline Eligibility confirmation* & & $\mathrm{x}$ & & & & & & & & & & & & \\
\hline $\begin{array}{l}\text { Pregnancy test and contraception } \\
\text { counselling } \dagger\end{array}$ & $x$ & $x$ & $x$ & $x$ & $x$ & $x$ & $x$ & $x$ & $x$ & $x$ & $x$ & $x$ & $x$ & $x$ \\
\hline Urine drug screening $\ddagger$ & $\mathrm{x}$ & $x$ & $x$ & $x$ & $x$ & $x$ & $x$ & $x$ & $x$ & $x$ & $x$ & $x$ & $x$ & $x$ \\
\hline Concomitant medication review & $x$ & $x$ & $x$ & $x$ & $x$ & $x$ & $x$ & $x$ & $x$ & $x$ & $x$ & $x$ & $x$ & $x$ \\
\hline Adverse events & & $x$ & $x$ & $x$ & $x$ & $x$ & $x$ & $x$ & $x$ & $x$ & $x$ & $x$ & $x$ & $x$ \\
\hline Dose adequacy & & $x$ & $x$ & $x$ & $x$ & $x$ & $x$ & $x$ & $x$ & $x$ & $x$ & $x$ & $x$ & $x$ \\
\hline Clinical Opiate Withdrawal Scale & & $x$ & $x$ & $x$ & $\begin{array}{l}\text { Wr } \\
\text { on }\end{array}$ & & & & ts & & & & & thdrawal, \\
\hline
\end{tabular}

\section{BUP-XR treatment}

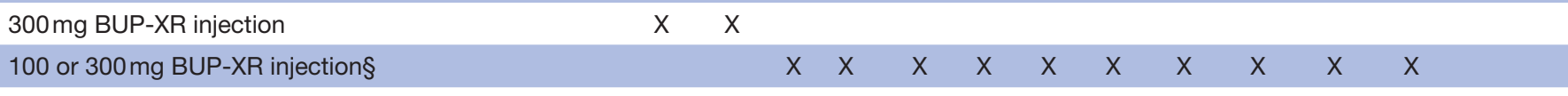

Telephone interviews

\begin{tabular}{|c|c|c|c|c|c|c|c|c|c|c|c|c|c|}
\hline Demographics & $x$ & & & & & & & & & & & & \\
\hline Subjective Opiate Withdrawal Scale & $x$ & & & $x$ & & & $\mathrm{x}$ & & & $\mathrm{x}$ & & & $x$ \\
\hline Opioid Craving Scale & $x$ & $x$ & $x$ & $x$ & $x$ & $\mathrm{x}$ & $x$ & $x$ & $x$ & $\mathrm{x}$ & $x$ & $x$ & $x$ \\
\hline ATOP & $x$ & $x$ & $x$ & $x$ & $x$ & $\mathrm{x}$ & $x$ & $x$ & $x$ & $x$ & $x$ & $x$ & $x$ \\
\hline Overdose (self-report) & $\mathrm{X}$ & $x$ & $\mathrm{X}$ & $x$ & $\mathrm{x}$ & $x$ & $\mathrm{x}$ & $x$ & $\mathrm{x}$ & $x$ & $x$ & $\mathrm{x}$ & $x$ \\
\hline Pain, Enjoyment, General Activity scale & $x$ & & & $x$ & & & $x$ & & & $x$ & & & $x$ \\
\hline Patient Health Questionnaire & $\mathrm{x}$ & & & $\mathrm{x}$ & & & $\mathrm{x}$ & & & $\mathrm{x}$ & & & $\mathrm{x}$ \\
\hline WHO Absenteeism and Presenteeism & $x$ & & & $x$ & & & $x$ & & & $x$ & & & $x$ \\
\hline $\begin{array}{l}\text { Treatment Satisfaction Questionnaire for } \\
\text { Medication }\end{array}$ & $\mathrm{x}$ & & & $\mathrm{x}$ & & & $x$ & & & $\mathrm{x}$ & & & $\mathrm{X}$ \\
\hline Early Cessation Questionnaire & & & & aso & & & & & & atm & & & \\
\hline
\end{tabular}

*Includes routine clinical tests where needed to confirm eligibility, forexample, suspected severe hepatic or renal impairment.

†ln women of childbearing potential.

$\ddagger$ At 3 selected sites, for validation of participant-reported drug use during interviews.

$\S F$ rom the $3^{\text {rd }}$ BUP-XR injection, the dose prescribed can be either 100 or $300 \mathrm{mg}$, at the discretion of the treating Investigator.

BUP-XR, extended-release buprenorphine; CoLAB, Community studies of Long-Acting Buprenorphine.

\section{Sample size}

Existing clients of participating general practice and public specialist drug and alcohol services will be approached and following informed consent will be screened for enrolment. A total of 100 participants will be enrolled and receive at least one dose of BUP-XR. The sample will allow estimation of the retention primary endpoint with adequate precision, and evaluation of factors impacting implementation at a variety of site types (specialist and primary care; public and private). Estimates of retention are based on phase III trials with BUP-XR products (estimated retention: $69 \%^{16} ; 95 \%$ CI $60.0 \%$ to $77.9 \%$ ).

\section{Data management}

Clinical data will be collected by participating sites and entered into a web-based electronic case report form, OpenClinica, an open-source clinical trial software for electronic data capture and clinical data management. 
Table 3 Primary and secondary endpoints of the CoLAB study

\section{Primary objective}

1. To examine extended-release buprenorphine BUP-XR)

treatment retention at 48 weeks

\section{Secondary objective}

1. To examine BUP-XR treatment retention and engagement in ongoing clinical care at 48 weeks

\section{Primary endpoint}

1.1 Proportion of participants retained in treatment at 48 weeks following initiation of monthly depot buprenorphine injections. Treatment retention is defined as remaining on active depot buprenorphine medication at 48 weeks.

Secondary endpoint

1.1 Proportion of participants retained in treatment at 48 weeks following initiation of monthly depot buprenorphine injections and engaged in ongoing clinical care. Treatment retention is defined as remaining on active depot buprenorphine medication AND completing a clinical assessment at 48 weeks.
2. To evaluate opioid craving, withdrawal, opioid and other drug use

\section{To evaluate client utilisation of buprenorphine} medication during the study, including BUP-XR dose variation, adherence with dosing schedule and dose supplementation

2.1 Change in clinically assessed (urinary drug screen) and clientreported use of opioids

2.2 Change in clinically assessed (urinary drug screen) and clientreported use of other drugs

2.3 Change in clinically assessed opioid craving

3.1 Percentage of participants who completed 12 injections (per protocol) during the 48 week study period.

3.2 Percentage of participants requiring dose adjustments with sublingual buprenorphine/buprenorphine-naloxone (and dose) during treatment

3.3 Percentage of participants maintained on $300 \mathrm{mg}$ per month and $100 \mathrm{mg}$ per month after the initial $2 \times 300 \mathrm{mg}$ injection 3.4 Mean duration of continuous treatment (weeks)

3.5 Reasons for drop-out among non-completers 3.6 Percentage of participants presenting to receive treatment within 7 and 14 days of the next scheduled injection 3.7 Mean duration (days) between administered injections

4. To evaluate treatment safety and tolerability by monitoring adverse events, and events of clinical interest such as drug-drug interactions and pain management in clients treated with BUP-XR
4.1 Percentage of participants with different types of 'special events of interest'

4.2 Percentage of participants with common adverse events (reported in greater than 5\%)

4.3 Percentage of participants with at least one severe or potentially life threatening (grade 3 or 4) adverse event;

4.4 Percentage of participants withdrawn from treatment due to unacceptable adverse events
5. To describe client-reported changes to health and social 5.1. Health service utilisation during treatment and estimated well-being

costs (including client travel)

5.2. Hours worked in paid employment/study

5.3. Other changes in health and social well-being (as measured by PEG, AQol-4D, ATOP client surveys)

6. To evaluate demographic, drug use and treatment factors associated with treatment outcomes

7. To evaluate client-reported experience of treatment

8. To examine BUP-XR treatment retention at 24 weeks

\section{To document the cost of the treatment at different \\ settings}

6.1 Demographic, drug use and treatment characteristics associated with treatment outcomes, for example, participant retention

\subsection{Client-reported treatment satisfaction measures}

8.1 Percentage of participants retained in treatment at 24 weeks following initiation of monthly depot buprenorphine injections. Treatment retention is defined as remaining on active depot buprenorphine medication at 24 weeks.

\subsection{Using process measures identify the resource use at both} client and facility level

AQoL-4D, Australian Quality of Life four-dimension; ATOP, Australian Treatment Outcome Profile; CoLAB, Community studies of Long-Acting Buprenorphine; PEG, Pain, Enjoyment, General Activity.

On-site monitoring and source data verification will be completed at regular intervals by the sponsor for data quality assurance and to ensure compliance with the study protocol, International Conference on Harmonisation Good Clinical Practice (ICH-GCP) guidelines, ethics approvals and sponsor standard operating procedures. 
Interviews will be conducted via telephone by UNSW researchers, not involved in the participants' clinical care or study team located at the site. Data will be entered by interviewers into the OpenClinica database. Multiple contact methods (eg, mobile, email, social media accounts) and those for participants' family or friends will be collected at baseline to facilitate intensive follow-up and maximise retention in research interviews.

\section{Qualitative research substudy}

The qualitative substudy aims to examine factors impacting BUP-XR implementation from the client, service provider, clinician and policy-maker perspective. Clients and providers will be interviewed to explore clients' interests, expectations and experiences of receiving BUP-XR treatment, providers' experiences of administering BUP-XR treatment in their service, and the organisational implications of implementing BUP-XR in service settings with varied characteristics in terms of model of care, staffing and policy. Qualitative interview data will be generated to examine factors that clients, service providers, clinicians and policy-makers perceive as important in the implementation and delivery of BUP-XR for the treatment of opioid dependence in community-based general practice and specialist treatment settings; how this new treatment is translated and made to work in its implementation contexts and practices; how the introduction of this treatment may impact treatment experience and shape service provision; and the possibilities of, and challenges posed by, this new treatment. Purposive sampling will be used to recruit consenting participants to participate in semistructured interviews, conducted either in person (where possible) or via telephone. Clients will be sampled to maximise diversity in gender, previous treatment experience and duration on BUP-XR, including those who discontinue treatment early. Interviews will capture a range of treatment timepoints including early BUP-XR experience soon after the first dose, and follow-up interviews after at least 6 months of treatment.

\section{Costing substudy}

A costing substudy will be undertaken to evaluate the impact of BUP-XR treatment on both participating services and participants. Resource use will be identified at both the client and facility level and will include process measures obtained through participant records (eg, medication charts), facility records (eg, appointment records) and interviews with individual clinical team members (eg, site PI, other treating staff and pharmacist) to document procedures related to drug storage, drug administration and client care. A bottom-up or activity-based costing will be used, where each of the resources used will be identified, measured and valued. This will include (but is not limited to) the implementation or start-up costs; staff training; treatment costs including time to prescribe, order/transport medication, dispense and administer; monitor drug storage (eg, refrigeration, temperature incursions and wastage); cost of the medication; any counselling or other healthcare costs, and any other consumables/supplies. Costs will be obtained from statebased salary and wage schedules, the Medicare Benefits Scheme (diagnostics, consultations etc), and other standard unit costs where relevant. Any relevant overhead and on-costs will be included. Monthly participant interviews will include collection of travel costs for BUP-XR treatment, changes in work hours, and health service utilisation unrelated to the facility providing BUP-XR.

\section{Patient and public involvement}

Design of the CoLAB implementation study was informed by a survey $(n=402)$ evaluating patients' priorities and concerns regarding BUP-XR. ${ }^{25}$ The research question, study design, participant information sheet and survey tools were reviewed by a community reference panel which included individuals with a history of or current drug use. Findings of the study will be disseminated through a lay language summary posted to participants, as well as publications distributed by advocacy agencies representing people who use drugs.

\section{DISCUSSION}

BUP-XR injections have been established as efficacious in licensing randomised controlled trials, ${ }^{15} 16$ however, further data are limited. ${ }^{27}$ The CoLAB study will provide data that reflect the 'real world' patient reported outcome and experience measures in a context as close to routine clinical care as possible, while also providing important data on costs, client and provider perspectives, and implementation facilitators and barriers. These data will further inform models of care, clinical guidelines and training programmes for health providers.

Although a recent survey conducted in Australia found that the majority of people who use opioids regularly anticipated that BUP-XR would be a good treatment option for them, ${ }^{25}$ criteria for effective BUP-XR client selection have not been established. One concern is that reduced clinician contact may impact treatment outcomes for some clients, through loss of daily structure and engagement with support services. Qualitative studies of potential clients found that 'longer' BUP-XR formulations (eg, monthly or 6 monthly) were viewed as beneficial for clients who wanted to avoid thinking about drugs and their networks of people who use drugs, wanted to reduce stigma, and desired 'normality' and 'recovery'. ${ }^{30}$ 'Shorter' BUP-XR formulations (eg, weekly) were viewed as beneficial to clients who were new to OAT, worried about the safety and reliability/effectiveness of OAT, want a 'break' from illicit opioids, and those who need more regular contact with services to monitor/support them. ${ }^{30}$ It is important to note, however, that the interest in a hypothetical treatment may not translate into actual experience and uptake of monthly BUP-XR injections.

Reduced frequency of clinic attendance also has implications for services providing OAT. While it may potentially free up resources for providing other services, the 
impact on clients' other healthcare needs during BUP-XR treatment is unknown. OAT for opioid dependence in Australia is incorporated into a broader treatment model of care involving regular clinical reviews, case management and psychosocial interventions, with services individualised for each client. ${ }^{1}$ In the CoLAB study, the minimum frequency of scheduled clinical reviews with a site investigator (or delegated clinician) is every 42 days. Participation in psychosocial services (eg, counselling) is encouraged, but not mandated, in the study protocol to more accurately reflect 'real world' clinical practice. The CoLAB study will provide important data on how clients receiving monthly BUP-XR injections use psychosocial and healthcare interventions in the periods between injections.

The study will also explore implementation issues related to management and administration of the BUP-XR medication. Sublocade must be stored and managed consistent with jurisdictional requirements for Schedule 8 (an Australian classification of drugs of dependence that are subject to additional regulatory controls regarding their manufacture, supply, distribution, possession and use ${ }^{31}$ ). Sublocade must also be stored under refrigerated conditions $\left(2^{\circ} \mathrm{C}-8^{\circ} \mathrm{C}\right)$ and is supplied via cold chain to participating clinics, however, is stable at ambient temperature for up to 7 days. The process of BUP-XR management, including distribution, receipt, storage and temperature control, permits and accountability in accordance with schedule 8 requirements will be documented to identify barriers. Sites with and without onsite schedule 8 compliant refrigerated storage have been included to examine these issues.

Although the study schedule of dosing and assessments is designed to allow flexibility and mimic 'real-world' clinical scenarios, there are many that will not be evaluated within the context of this research protocol. For example, all participants must be stable on a daily dose of $8-32 \mathrm{mg}$ sublingual buprenorphine for at least 7 days immediately prior to the first BUP-XR injection. The study will, therefore, not provide answers to clinical questions such as initiation of Sublocade in patients using methadone or illicit heroin, treatment of clients on low sublingual buprenorphine doses (less than $8 \mathrm{mg}$ daily) with BUP$\mathrm{XR}$, and transfer of clients between the two current BUP-XR products (Sublocade and Buvidal). Clinical guidelines regarding these specific scenarios have been developed in Australia, ${ }^{31}$ with recommendations based on the available evidence and expert consensus, though further research is required. A further limitation is the single arm design. The relatively small sample size and lack of comparator limits the ability to evaluate effectiveness and cost-effectiveness of BUP-XR compared with the sublingual buprenorphine standard of care; however, the CoLAB study will provide important information on the client retention, tolerability and acceptability of BUP$\mathrm{XR}$, as well as the cost and key considerations involved in integrating this formulation into clinical practice in Australia.
The introduction of depot buprenorphine formulations is likely to have significant benefits for some clients and their service providers. BUP-XR may not suit all OAT clients, and some will prefer methadone or sublingual buprenorphine treatment. Current treatment guidelines recommend that medication choice is guided by client factors such as prior experience with medications, adverse events, drug-drug interactions, overdose risks and in some cases logistic factors such as travel requirements. It is also possible that prescribers may prefer BUP-XR where there are concerns regarding a client's non-medical use (eg, injecting, hoarding, diversion to others) of sublingual buprenorphine, or a client has a number of risk factors for unsupervised dosing that are difficult to mitigate, such as homelessness, high-risk substance use, or history of medication diversion. Uptake and experience of clients will also be impacted by the way in which the medication is incorporated into existing OAT policies $\left(\mathrm{eg},{ }^{31}\right)$, education on delivering BUP-XR for healthcare professionals, communication strategies and engagement of consumer groups and peers. The CoLAB study will provide important data to inform these activities.

\section{ETHICS AND DISSEMINATION}

The study is sponsored and managed by the National Drug and Alcohol Research Centre (NDARC), UNSW Sydney, Australia. Oversight is provided by a PSC composed of clinical specialists in addiction medicine, epidemiologists with expertise in opioid dependence and drug user research, qualitative researchers, health economist, NDARC research and clinical trials operational staff and representatives from participating clinic sites. The protocol was informed by a survey of over 400 consumers evaluating attitudes and preferences for OAT. The PSC includes representatives of community organisations advocating for people who use drugs. This study has received ethics approval from the St Vincent's Hospital Sydney Human Research Ethics Committee (Ref. HREC/18/SVH/221), and site specific assessments have been approved by the local research governance offices of participating sites. Future amendments will be similarly submitted for approval. The study will be conducted in accordance with ICH-GCP guidelines, the Declaration of Helsinki, and all applicable local ethical and regulatory requirements. All participants must provide written informed consent. The findings will be disseminated via publication in a peer-reviewed journal and relevant scientific conference presentations. In addition to the primary clinical study report, we aim to publish a series of secondary papers describing the various components of the study, including implementation issues, qualitative and costing substudies, and clinical guidance development. The authors will disseminate findings to the affected community by engaging organisations representing users.

Author affiliations

${ }^{1}$ National Drug and Alcohol Research Centre, UNSW Sydney, Sydney, New South Wales, Australia 
${ }^{2}$ School of Psychology, University of Wollongong, Sydney, New South Wales, Australia

${ }^{3}$ Illawarra Health and Medical Research Institute, University of Wollongong, Wollongong, New South Wales, Australia

${ }^{4}$ The Kirby Institute, UNSW Sydney, Sydney, New South Wales, Australia ${ }^{5}$ Discipline of Addiction Medicine, University of Sydney, Surry Hills, New South Wales, Australia

${ }^{6}$ The Langton Centre, South East Sydney Local Health District, Surry Hills, New South Wales, Australia

${ }^{7}$ Monash Addiction Research Centre and Eastern Health Clinical School, Monash University Peninsula Campus, Frankston, Victoria, Australia

${ }^{8}$ School of Population and Global Health, University of Melbourne, Melbourne, Victoria, Australia

${ }^{9}$ Centre for Social Research in Health, UNSW Sydney, Sydney, New South Wales, Australia

${ }^{10}$ Faculty of Health and Medical Sciences, University of Adelaide, Adelaide, South Australia, Australia

Twitter Suzanne Nielsen @drsuzinielsen and Jason Grebely @jasongrebely

Acknowledgements Study medication is provided by Indivior. The authors would also like to thank all the participants in the study, the study staff, clinical staff, and staff in alcohol and other drug services for supporting this research.

Collaborators The CoLAB Study Team are Michael Farrell (Chief Investigator; PSC), Louisa Degenhardt (PSC), Nicholas Lintzeris (Chief Medical Officer; PSC), Briony Larance (PSC), Suzanne Nielsen (PSC), Jason Grebely (PSC), Gregory Dore (PSC), Robert Ali (PSC), Kari Lancaster (PSC), Marian Shanahan (PSC), Carla Treloar (PSC), Marianne Byrne (Clinical Trials Manager; PSC), Jeyran Shahbazi (Trial Coordinator; PSC), Stella Nalukwago (health economic analyses), Craig Rodgers (Site Investigator; PSC), Adrian Dunlop (Site Investigator; PSC), Michael McDonough (Site Investigator), Jon Cook (Site Investigator), Mark Montebello (Site Investigator), Michael Aufgang (Site Investigator) and Robert Weiss (Site Investigator), Madeline News (interview data collection), Zoe Griffin (research data collection).

Contributors BL, SN, MS, LD, KL, JG, GD, RA and MF conceived the original study concept, contributed to the study design and obtained funding. BL, NL, MB, JS, SN, MS, LD, JG and MF participated in survey development, design of data collection and contributed to the detailed trial protocol. MB and BL drafted the manuscript with input from all authors. All authors meet the International Committee of Medical Journal Editors criteria for authorship and have read and approved the final manuscript.

Funding This study is sponsored by the National Drug and Alcohol Research Centre, University of New South Wales, Sydney Australia. The Study is supported by an Externally Sponsored Collaborative Research grant from Indivior. Indivior contributed to the study design and analysis plan. Indivior is supplying its BUP-XR product, Sublocade, for all study participants.

Disclaimer Indivior will have no role in collection, analysis and interpretation of data; in the writing of the manuscript; or in the decision to submit the manuscript for publication.

Competing interests Many of the investigators have received investigatorinitiated, untied educational grants for opioid-related studies from: Indivior (Briony Larance, Louisa Degenhardt, Nicholas Lintzeris, Michael Farrell, Suzanne Neilsen); Reckitt Benckiser (Briony Larance, Louisa Degenhardt, Nicholas Lintzeris, Michael Farrell, Suzanne Nielsen, Robert Ali, Adrian Dunlop); Mundipharma (Briony Larance, Louisa Degenhardt, Nicholas Lintzeris, Michael Farrell); and Seqirus (Briony Larance, Louisa Degenhardt, Michael Farrell, Suzanne Nielsen).

Patient consent for publication Not required.

Provenance and peer review Not commissioned; externally peer reviewed.

Open access This is an open access article distributed in accordance with the Creative Commons Attribution Non Commercial (CC BY-NC 4.0) license, which permits others to distribute, remix, adapt, build upon this work non-commercially, and license their derivative works on different terms, provided the original work is properly cited, appropriate credit is given, any changes made indicated, and the use is non-commercial. See: http://creativecommons.org/licenses/by-nc/4.0/.

\section{ORCID iDs}

Marianne Byrne http://orcid.org/0000-0002-3489-2532

Suzanne Nielsen http://orcid.org/0000-0001-5341-1055

Louisa Degenhardt http://orcid.org/0000-0002-8513-2218
Marian Shanahan http://orcid.org/0000-0001-9873-3576

\section{REFERENCES}

1 Gowing L, Ali R, Dunlop A, et al. National guidelines for medicationassisted treatment of opioid dependence. Canberra: Commonwealth of Australia, 2014

2 Rhodes T. The becoming of methadone in Kenya: how an intervention's implementation constitutes recovery potential. Soc Sci Med 2018;201:71-9.

3 Vuong T, Shanahan M, Nguyen N, et al. Cost-Effectiveness of CenterBased compulsory rehabilitation compared to community-based voluntary methadone maintenance treatment in HAI Phong City, Vietnam. Drug Alcohol Depend 2016;168:147-55.

4 Independent Expert Working Group. Clinical guidelines on drug misuse and dependence update 2017. drug misuse and dependence: UK guidelines on clinical management. London: Department of Health, 2017.

5 Madden ALT, Bath N, Winstock AR. Satisfaction guaranteed? what clients on methadone and buprenorphine think about their treatment. 2008;27:671-8.

6 Rowe J. A raw deal? impact on the health of consumers relative to the cost of pharmacotherapy. Melbourne: RMIT University, Centre for Applied Social Research, 2007.

7 Albayaty M, Linden M, Olsson $\mathrm{H}$, et al. Pharmacokinetic evaluation of once-weekly and Once-Monthly buprenorphine subcutaneous injection depots (CAM2038) versus intravenous and sublingual buprenorphine in healthy volunteers under naltrexone blockade: an open-label phase 1 study. Adv Ther 2017;34:560-75.

8 Haasen C, Linden M, Tiberg F. Pharmacokinetics and pharmacodynamics of a buprenorphine subcutaneous depot formulation (CAM2038) for once-weekly dosing in patients with opioid use disorder. J Subst Abuse Treat 2017;78:22-9.

9 Camurus Pty Ltd. Australian product information. Buvidal Weekly and Buivdal monthly (buprenorphine) 2018.

10 Lintzeris N, Dunlop A, Masters D. Clinical guidelines for use of depot buprenorphine (Buvidal ${ }^{\circledR}$ and Sublocade) in the treatment of opioid dependence. Sydney, Australia: NSW Ministry of Health, 2019.

11 Indivior Inc. Australian product information. Sublocade 2019.

12 Laffont CM, Gomeni R, Heidbreder C, et al. Population pharmacokinetic modeling after repeated administrations of RBP6000 , a new, subcutaneously injectable, long-acting, sustainedrelease formulation of buprenorphine, for the treatment of opioid use disorder. J Clin Pharmacol 2016;56:806-15.

13 Nasser AF, Greenwald MK, Vince B, et al. Sustained-Release buprenorphine (RBP-6000) blocks the effects of opioid challenge with hydromorphone in subjects with opioid use disorder. J Clin Psychopharmacol 2016;36:18-26.

14 Walsh SL, Comer SD, Lofwall MR, et al. Effect of buprenorphine Weekly depot (cam2038) and hydromorphone blockade in individuals with opioid use disorder: a randomized clinical trial. JAMA Psychiatry 2017;74:894-902.

15 Haight BR, Learned SM, Laffont CM, et al. Efficacy and safety of a monthly buprenorphine depot injection for opioid use disorder: a multicentre, randomised, double-blind, placebo-controlled, phase 3 trial. Lancet 2019;393:778-90.

16 Lofwall MR, Walsh SL, Nunes EV, et al. Weekly and monthly subcutaneous buprenorphine depot formulations vs daily sublingual buprenorphine with naloxone for treatment of opioid use disorder: a randomized clinical TrialEfficacy of subcutaneous Weekly and monthly vs daily sublingual dosing of buprenorphine for opioid use disorder. JAMA Internal Medicine 2018;178:764-73.

17 US Food and Drug Administration. Drug approval package: SUBLOCADE (buprenoprhine), 2017. Available: https:// www.accessdata.fda.gov/drugsatfda_docs/nda/2017/ 209819Orig1s000TOC.cfm [Accessed 15 Aug 2019].

18 Sublocade buprenorphine. Therapeutic goods administration. public ARTG summary, 2019. Available: http://tga-search.clients. funnelback.com/s/search.html?query=sublocade\&collection=tgaartg\&profile=record [Accessed 15 Aug 2019].

19 Therapeutic Goods Administration. Public ARTG summary: Buvidal buprenorphine, 2019. Available: http://tga-search.clients.funnelback. $\mathrm{com} / \mathrm{s} /$ search.html?query=buvidal\&collection=tga-artg\&profile= record [Accessed 15 Aug 2019].

20 European Medicines Agency. Buvidal marketing Authorisation details, 2018. Available: https://www.ema.europa.eu/en/medicines/human/ EPAR/buvidal\#authorisation-details-section [Accessed 15 Aug 2019].

21 Rosenthal RN, Goradia VV. Advances in the delivery of buprenorphine for opioid dependence. Drug Des Devel Ther 2017;11:2493-505. 
22 Larance B, Degenhardt L, Lintzeris N, et al. Post-Marketing surveillance of buprenorphine-naloxone in Australia: diversion, injection and adherence with supervised dosing. Drug Alcohol Depend 2011;118:265-73.

23 Larance B, Lintzeris N, Ali R, et al. The diversion and injection of a buprenorphine-naloxone soluble film formulation. Drug Alcohol Depend 2014;136:21-7.

24 Lofwall MR, Walsh SL. A review of buprenorphine diversion and misuse: the current evidence base and experiences from around the world. J Addict Med 2014;8:315-26.

25 Larance B, Degenhardt L, Grebely J, et al. Perceptions of extendedrelease buprenorphine injections for opioid use disorder among people who regularly use opioids in Australia. Addiction 2019 doi:10.1111/add.14941. [Epub ahead of print: 20 Dec 2019].

26 Burns L, Gisev N, Larney S, et al. A longitudinal comparison of retention in buprenorphine and methadone treatment for opioid dependence in New South Wales, Australia. Addiction 2015;110:646-55.
27 Frost M, Bailey GL, Lintzeris N, et al. Long-Term safety of a Weekly and monthly subcutaneous buprenorphine depot (CAM2038) in the treatment of adult out-patients with opioid use disorder. Addiction 2019;114:1416-26.

28 Indivior Inc. Investigator's brochure. RBP-6000 (buprenorphine in the ATRIGEL delivery system. Richmond, USA: Indivior, 2017.

29 Indivior Inc. Investigator's brochure. buprenorphine (extendedrelease injection) for subcutaneous use. North Chesterfield, USA: Indivior, 2018.

30 Neale J, Tompkins CNE, Strang J. Prolonged-release opioid agonist therapy: qualitative study exploring patients' views of 1 -week, 1-month, and 6-month buprenorphine formulations. Harm Reduct $J$ 2019;16:25.

31 Therapeutic Goods Administration. Standard for the uniform scheduling of medicines and poisons (SUSMP). Australian Government Department of Health and Ageing, 2013. 